\title{
Low level light therapy for the treatment of recalcitrant chalazia: a sample case summary
}

This article was published in the following Dove Press journal:

Clinical Ophthalmology

\section{Karl Stonecipher' \\ Richard Potvin (1D)}

'Physicians Protocol, Greensboro, NC, USA; ${ }^{2}$ Science in Vision, Akron, NY, USA
Purpose: To evaluate the effects of low-level light therapy (LLLT) on the resolution of recalcitrant chalazia.

Patients and Methods: This was a single-site retrospective chart review of patients with chalazia, all of whom were unresponsive to previous pharmaceutical therapy or surgical intervention, who received a 15 min LLLT treatment in conjunction with a standard pharmaceutical regimen. A second treatment was applied $24 \mathrm{hrs}$ to as late as 2 months if there was no evidence of progression of resolution in appearance.

Results: A total of 26 eyes of 22 patients with relevant history and treatment were reviewed, all with a history of prior pharmaceutical treatment for their chalazia. After a single 15 min LLLT treatment, followed by a standard pharmaceutical regimen, $46 \%$ of eyes $(12 / 26)$ showed resolution of their chalazia. Resolution was noted from 3 days to one-month posttreatment. With a second treatment, the chalazia resolved in $92 \%$ of eyes $(24 / 26)$. Only two eyes of the $26(8 \%)$ required incision and curettage after LLLT treatment.

Conclusion: The use of LLLT for the treatment of recalcitrant chalazia appears to be beneficial in patients who have failed topical and/or systemic therapy, significantly reducing the likelihood of requiring surgical intervention.

Keywords: LLLT, low level light therapy, chalazion, chalazia

\section{Plain language summary}

A chalazion (plural chalazia) is an eyelid cyst that gives the eyelid a swollen appearance. Chalazia are the result of a plugged meibomian gland, the glands in your eyelids that produce meibum, the oil necessary for good tears. Contents of the gland solidify and restrict the normal flow of meibum, resulting in swelling.

Often warm compresses or drugs are used to treat chalazia. Resolution may take weeks or months. Some individuals have chalazia that recur often, or never resolve, even with such treatment. At that point a minor surgery may be required, cutting the eyelid open to remove the hardened material in the gland.

A new light-based treatment which has recently been shown to be beneficial for dry eye related to meibomian gland problems was tested in the current study, to see if it could help patients with chalazia. The treatment involved using low level red light applied to the upper face (the cheek and over the closed lids). Treatment was administered to patients who had chalazia that did not respond to warm compresses and/or drug therapy or surgery.

In 9 of the 19 patients treated, a single 15 min session was enough to promote resolution

Correspondence: Karl Stonecipher Physicians Protocol, 1002 North Church Street, Suite I0I, Greensboro, NC 27408, USA

Tel +I 3362888823

Email Stonenc@gmail.com of the chalazion. A second treatment was applied to 10 patients, 8 of whom had resolution of their chalazia. Only two cases required surgical intervention. It may be concluded that low level light therapy can promote resolution of chalazia, significantly reducing the percentage of eyes requiring surgery. 


\section{Introduction}

The most common benign granulomatous inflammatory lid masses of the eye are chalazia. ${ }^{1}$ The main etiology behind the formation of a chalazion is inflammation of the meibomian gland and inspissation of the meibum in the gland. ${ }^{1}$ Chalazia are generally self-limiting but may become chronic and recur, with patients complaining of a non-tender lid mass that has slowly enlarged over weeks or months. ${ }^{1}$ Chalazia can take several months to resolve on their own, ${ }^{2}$ with an older study noting that $25 \%$ may resolve on their own over an average period of 6 months. ${ }^{3}$ However, chalazia are generally treated when noted, because they may grow big enough to impair vision or become infected, resulting in possible cellulitis. ${ }^{1}$

The most common non-surgical and surgical treatment options for chalazia, from least invasive to most invasive, are warm compresses, topical medications (i.e., antibiotic, corticosteroid or antibiotic corticosteroid combinations), systemic medications (i.e., oral antibiotics such as tetracycline or doxycycline), steroid injection or surgical incision and curettage (I\&C). Warm compresses or therapeutic masks offer the lowest complication rate but also the least success; this may be due to the high dependence of the procedure being performed by the patient at home in a non-controlled setting. The most invasive treatment is the I\&C surgical procedure. It has been demonstrated to be more effective than steroid injection alone after one treatment. ${ }^{4}$ It is generally effective but can result in lid notching if the lid margin is involved, or eyelid perforation with resultant scarring and hemorrhaging. Globe perforation is also possible with both I\&C and steroid injection. ${ }^{2}$ Given that current treatments either have low success or carry some risk of complications, new non-surgical treatment approaches are of interest.

One potential candidate for non-surgical treatment includes the use of low-level light therapy or LLLT; this is a form of photobiomodulation that was developed for dermatological and other medical uses. The light is considered "low-level" relative to lasers used to ablate tissue because the fluence is lower than would be required to generate a cellular thermal effect. Low-level laser biostimulation was first noted to have a positive therapeutic effect in $1968 .^{5}$ While lasers are coherent light sources, non-coherent light (such as from light emitting diodes, or LEDs) has been shown to have a similar effect. One feature of LED light is photon interference, which increases photon intensity and penetration below the skin. Light emitting diodes (LEDs) of specific wavelengths (often $590-633 \mathrm{~nm}$ ) have consistently demonstrated a beneficial effect on skin cells. ${ }^{6,7}$ Effects on non-skin cells have also been demonstrated, with one example being decreased muscle fatigue associated with red LLLT therapy. ${ }^{8}$ While the specific mechanism of action for LLLT has not been identified, cellular photoactivation is presumed. ${ }^{9}$ The effect may be related to activation of mitochondria, ${ }^{10}$ vascular endothelial growth factor $(\mathrm{VEGF})^{11}$ or anti-inflammatory activity through the regulation of reactive oxygen species. ${ }^{12}$ There is also a warming effect associated with LLLT, though not a thermal effect sufficient to disrupt tissue.

Recent studies have demonstrated the value of red-light LLLT in the treatment of meibomian gland disease. One study showed significant improvement in OSDI scores, tear breakup time and meibomian gland grade after treatment with a combination of intense pulsed light and LLLT. $^{13}$ Another showed significant increases in tear breakup time after treatment with red light. ${ }^{14}$ Because these improvements are presumed to be related to overall improvements in cellular function as described above, there is a reasonable likelihood that chalazia might better resolve under these improved metabolic conditions.

The current case series was collected to investigate the effects of LLLT on the resolution of chalazia that did not respond to prior non-surgical or surgical treatment. The LLLT device investigated here was part of the Eye-light ${ }^{\circledR}$ (outside the USA), or Epi-C PLUS system (in the USA), both from Espansione Marketing S.p.A., Bologna, Italy. These units include the ability to deliver both Intense Pulsed Light (IPL) and LLLT. The Epi-C PLUS unit is approved for dermatological use in the USA. The lowlevel light source for this device is a matrix of LEDs with a wavelength of $633 \pm 10$ nanometers and an emission power of $100 \mathrm{~mW}$ per $\mathrm{cm}^{2}$. When a $15 \mathrm{~min}$ treatment is applied, the total fluence in the treated area is 110 Joules per $\mathrm{cm}^{2}$.

\section{Patients and methods}

This retrospective chart review was approved by an institutional review board (Salus IRB, Austin, TX), which also granted a waiver of informed consent for use of patients' de-identified clinical data. As this was a retrospective review, no clinical registration (e.g., clinicaltrials.gov) was required. Written informed consent to use the photos in this manuscript was obtained from the guardian (Figure 1) and patient (Figure 2). The chart review included data from one physician at one site. Patient demographics, prior history related to 


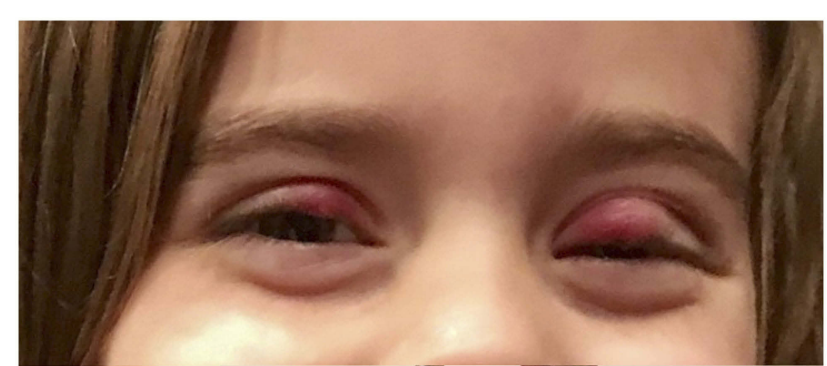

A

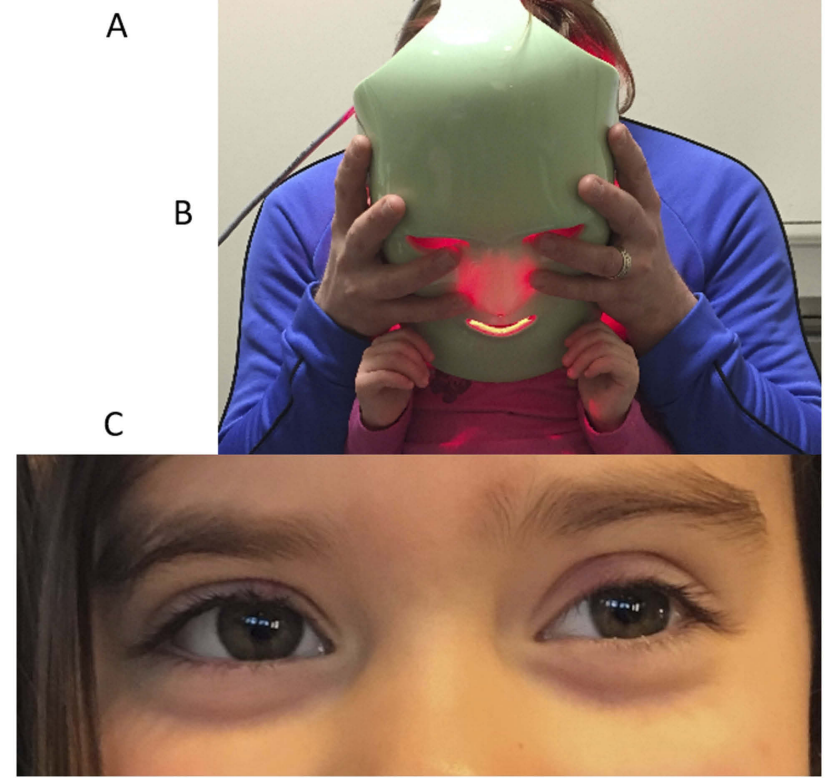

Figure I A young patient before LLLT treatment $(\mathbf{A})$, with treatment being applied (B) and I week after treatment (C). Images used with permission.

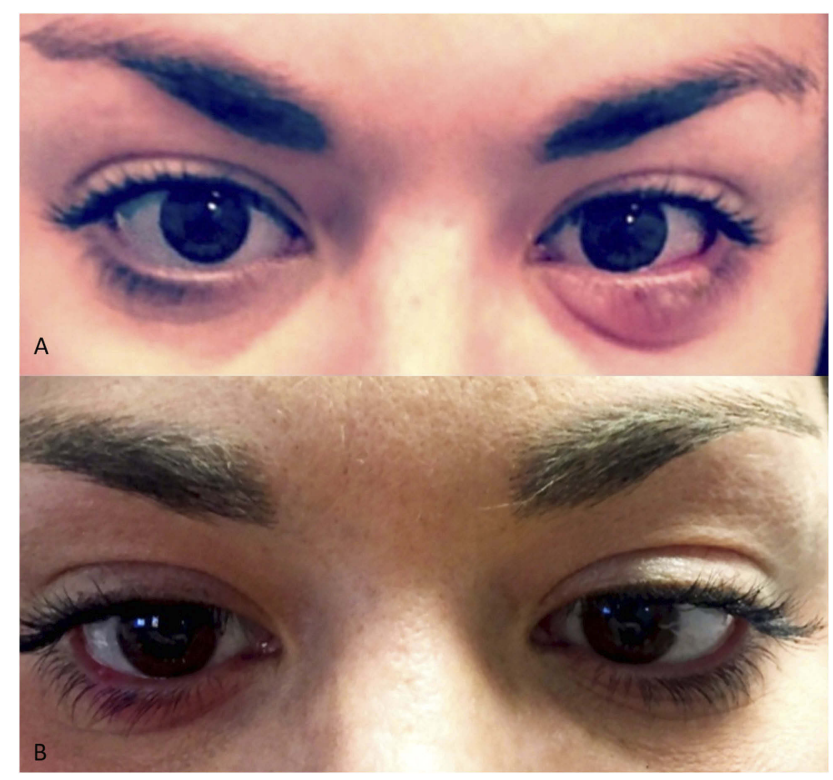

Figure 2 An adult patient before LLLT treatment (A), and 2 weeks after treatment (B). Images used with permission.

chalazia and treatment specifics were extracted from the file, along with the results of the treatment.
All patients were referred for treatment after several unsuccessful attempts at other pharmaceutical treatments, either topical or oral. One patient in the series had had surgical intervention prior to presentation, a standard I\&C, with no long-term resolution. Each patient received Low Level Light Therapy (LLLT) for 15 mins delivered via the Epi-C Treatment mask at one sitting. After the LLLT treatment patients received an additional round of antibiotic/steroid eye drops for two weeks. All adult patients also received Doxycycline $100 \mathrm{mg}$ by mouth twice per day; patients under the age of thirteen did not receive oral therapy. If there was no apparent change in the chalazion at the $24-48 \mathrm{hr}$ visit, a second $15 \mathrm{~min}$ treatment was considered. Second treatments were performed from $24 \mathrm{hrs}$ to 2 months after the initial treatment. All patients were followed to resolution, defined as no visible sign of the chalazion post-treatment.

\section{Results}

A total of 26 eyes of 22 patients with relevant history and treatment were identified in the clinical records. Of these, all had prior pharmaceutical treatment; previous I\&C had been performed on one patient. Prior treatments typically involved some combination of antibiotics, an antibiotic/steroid combination and/or oral doxycycline. Table 1 summarizes the relevant pre-treatment and post-treatment data for each patient.

As can be seen, $46 \%$ of eyes (12/26) required only one 15 min LLT treatment with the prescribed pharmaceutical regimen to resolve the chalazion; resolution was noted from 3 days to one-month post-treatment. Ten patients (14 eyes) received a second treatment within two months of the first. In all but two of these patients (two eyes) the chalazion resolved. The overall success rate with LLLT treatment was therefore $92 \%(24 / 26)$. There was no observable pattern in terms of who might need a second treatment, though the two youngest patients were both treated twice; neither of these patients received systemic treatment or surgical intervention.

Only two eyes of the $26(8 \%)$ required incision and curettage after LLLT treatment. One eye of one patient received an intralesional corticosteroid at the time of I\&C.

Figure 1A shows a young patient with a significant chalazion on the upper left lid, and some notable swelling on the other lids. She had failed multiple non-surgical options, including warm compresses, topical antibiotic/steroid drops and topical antibiotic ointments. Figure 1B shows the LLLT treatment mask being applied to the face of this 
Table I List of patients treated

\begin{tabular}{|c|c|c|c|c|c|c|c|c|c|}
\hline ID & Age & Eye & $\begin{array}{l}\text { Upper or } \\
\text { Lower Lid }\end{array}$ & $\begin{array}{l}\text { Prior Tx } \\
\text { history }\end{array}$ & Treatment & $\begin{array}{l}\text { Second } \\
\text { Treatment }\end{array}$ & $\begin{array}{l}\text { Time between } \\
\text { treatments }\end{array}$ & $\begin{array}{l}\text { Resolution } \\
\text { after LLLT }\end{array}$ & $\begin{array}{l}\text { Time to } \\
\text { Resolution }\end{array}$ \\
\hline I & 49 & OD & Upper & $\begin{array}{l}\text { KENALOG/ } \\
\text { DCN/ABXS }\end{array}$ & I & 0 & & Yes & I week \\
\hline 2 & 75 & OD & Upper & I\&C & I & 0 & & Yes & I week \\
\hline 3 & 21 & OD & Upper & DCN/ABXS & I & 0 & & Yes & I month \\
\hline 4 & 39 & OD & Upper/lower & DCN/ABXS & I & 0 & & Yes & I week \\
\hline 5 & 61 & OD & Lower & $D C N / A B X S$ & I & 0 & & Yes & 3 days \\
\hline 6 & 52 & OD & Upper & $\begin{array}{l}\text { DCN/ABXS } \\
\times 2\end{array}$ & I & 0 & & Yes & I month \\
\hline 7 & 33 & os & Lower & $A B X / A B X S$ & I & 0 & & Yes & 2 weeks \\
\hline 8 & 71 & OD & Upper & $A B X S$ & I & 0 & & Yes & I week \\
\hline 9 & 27 & OS & Upper & $A B X S$ & I & 0 & & Yes & I week \\
\hline 10 & 71 & OS & LUL & ABSX & I & 0 & & Yes & 3 days \\
\hline 11 & 31 & OD & LLL & ABSX & I & 0 & & Yes & I week \\
\hline 12 & 93 & OD & RLL & $A B X S$ & I & 0 & & Yes & I week \\
\hline 13 & 60 & OD & Lower & $D C N / A B X S$ & I & I & $24 \mathrm{hrs}$ & Yes & I week \\
\hline 14 & 53 & OD & Upper & $\begin{array}{l}\mathrm{DCN} / \mathrm{ABXS} \\
\mathrm{X} 2\end{array}$ & I & I & 2 months & No & \\
\hline 15 & 47 & OD & Upper & DCN/ABXS & I & I & I month & Yes & I month \\
\hline 16 & 6 & OU & Upper/lower & $A B X / A B X S$ & I & I & 2 months & Yes & 10 weeks \\
\hline 17 & 4 & OU & upper/lower & $A B X / A B X S$ & I & I & 2 months & Yes & 9 weeks \\
\hline 18 & 4 & OU & upper/lower & $A B X / A B X S$ & I & I & 2 months & Yes & 10 weeks \\
\hline 19 & 6 & OU & Upper/lower & $A B X / A B X S$ & I & I & 2 months & Yes & 10 weeks \\
\hline 20 & 35 & OD & Upper & $A B X S$ & I & I & $24 \mathrm{hrs}$ & Yes & 3 days \\
\hline 21 & 51 & OD & Upper & $A B X S$ & I & I & 7 days & No & \\
\hline 22 & 71 & OS & Lower & $A B X S$ & I & I & 2 months & Yes & 10 weeks \\
\hline
\end{tabular}

Abbreviations: $\mathrm{DCN}$, oral doxycycline; I\&C, incision and curettage; $A B X$, antibiotic; $A B X S$, antibiotic/steroid combination.

patient, being held on her mother's lap. Figure 1C shows the results one week after LLLT treatment; significant improvement is evident.

In another example, Figure 2A shows an adult patient with a chalazion on the lower left lid. As with the previous patient, she had been treated in the past with warm compresses, topical antibiotic/steroid drops, topical antibiotic ointments. She had also received oral doxycycline prior to the LLLT treatment without resolution. Figure 2B shows the same patient two weeks after her LLLT treatment.

\section{Discussion}

The authors reviewed LLLT treatment of chalazia since 2017 in one practice in patients ranging in age from 4 to 93 years old. To the best of our knowledge, this is the first series of patients reported in the literature.

As noted earlier, alternative treatment options range from warm compresses to surgical excision. A randomized multicenter clinical trial of 105 patients (141 cases), found $18 \%$ (with an additional $30 \%$ lost to follow-up presumably due to chalazia resolving) had complete resolution after warm compresses treatment with or without the addition of topical antibiotic or antibiotic steroid combination; despite similar outcomes, patients had higher satisfaction if they were given prescription medication. ${ }^{15}$ Of the cases that completely resolved, $86 \%$ resolved within 6 months; chalazia that resolved fully took less time than those which partially resolved (1.5 vs 2.2 months). ${ }^{15}$ If chalazia were present for greater than 2 months, then they were more likely to benefit from the initial procedure being more invasive. ${ }^{15}$ Jackson et al noted that treatment success was $29 \%$ with warm compresses and chloramphenicol ointment within 3 months versus $72 \%$ after I\&C. ${ }^{16}$ Steroid injections offer advantages over surgical excision since they can be used for multiple lesions and for chalazia close to the punctum to avoid injury and potential epiphora as well as when the patient is less cooperative such as in a pediatric population. ${ }^{17}$ Steroid injections using triamcinolone acetonide are usually administered directly to the lesion. ${ }^{18}$ Intralesional steroid injection reduced chalazia 
size by more than $80 \%$ in $60 \%$ of patients after one injection and $20 \%$ of patients after two injections over an average time of 2.5 weeks with no difference between new and recurrent cases; if significant improvement was not noted after two injections then surgical intervention appears more suitable. ${ }^{18}$

A 2016 meta-analysis compared steroid injection with $\mathrm{I} \& \mathrm{C}$ and concluded that the surgical intervention was more effective after one procedure $(60 \%$ vs $78 \%)$ with a decrease in relative efficacy if taking into account a second attempt (73\% vs 87\%); there were no differences in complication rates between the two procedures. ${ }^{4}$ Singhania et al noted that the resolution of medium to large size chalazia (chalazia diagnosis was confirmed by biopsy) was comparable between one steroid injection (90\% for medium and 74\% for large) and I\&C (92\% for medium and $91 \%$ for large) taking an average of 2-3 weeks to resolve; the reason for the better results in the steroid group may be attributed to removal of material within the lesion for biopsy before steroid injection. ${ }^{19}$ In a study of 536 patients, I\&C resulted in satisfactory outcome in $91 \%$ of cases though chalazia recurred in $6 \%$ of cases. ${ }^{20}$ Goawalla et al compared the three treatment modalities and noted no difference in outcome between steroid and surgical interventions ( $84 \%$ and $87 \%$, respectively) with a lower success rate $(46 \%)$ using compresses; pain scores were highest for the surgical group, inconvenience was least in the steroid group and satisfaction was lowest in the compresses group. ${ }^{21}$

Adverse events with such common treatments have been reported. McMonnies et al note that the combination of heat and eye massage, such as when patients are prescribed warm compresses, could result in corneal distortion and may warrant follow-up using corneal topography. ${ }^{22}$ One downside to more conservative treatments (anything aside from surgical excision) is the potential to mask, delay or miss a more concerning diagnosis such as a cancerous lesion that requires biopsy. Steroid injections also carry an additional risk if used in an infected eye ${ }^{17}$ as they can increase intraocular pressure or result in depigmentation of the $\operatorname{skin}^{2}$ or vascular occlusion with loss of vision. ${ }^{23}$ Botulinum toxin complications include hematoma, ptosis, keratoconjunctivitis sicca, epiphora, reduced blink rate but all are expected to resolve once the paralysis effects of the injection wear off. ${ }^{24}$

Other less common ways to manage chalazia include vitamin A supplements or botulinum toxin injections. ${ }^{24,25}$ Vitamin $\mathrm{A}$ is expected to work since the changes in chalazia are consistent with changes seen in Vitamin A deficiency; only one report has been documented so more research is warranted in this area. ${ }^{25}$ Botulinum toxin mechanism in treating chalazia is by decreasing meibomian gland secretion and Knezevic et al noted that in a placebo-controlled study, one injection of botulinum toxin was sufficient in $81 \%$ of subjects with an average of about 2.5 weeks for chalazia to resolve. ${ }^{24}$

The advantage of LLLT over these other therapies is that it is non-surgical, can be performed in the office by a technician and the risk of side-effects is negligible. It can be used without regard to race and/or skin type and can reduce the risk of exposure to antibiotic therapy in the pediatric population. There are also no contraindications to a second treatment, if necessary. The high success rate of LLLT treatment achieved in this sample population $(92 \%$ resolution by 2 months after one or two treatments) is notable.

There are limitations to the current study. LLLT treatment was always combined with a pharmaceutical regimen, to maximize the likelihood of resolution of the chalazion for patients. The pediatric patients were treated without additional systemic intervention to reduce potential complications related to oral antibiotic therapy. As such, no information related to LLLT treatment alone is available. A controlled trial to investigate the effects of LLLT alone or in combination with pharmaceutical therapy is of future interest. In addition, there was no specific grading of the extent or appearance of the chalazia treated. As such, no specific comments can be made as to whether a certain presentation is more amenable to LLLT treatment. Finally, the patient set reported here were those patients with chalazia that had failed with prior treatment. These might be considered a subset of patients presenting with chalazia. However, the primary author (KS) notes that he has had similar success with patients presenting with chalazia for the first time; all 11 patients he treated in the same time period had their chalazia resolve in 3 weeks or less (these were pediatric and adult patients ranging in age from 3 to 59 years old).

\section{Conclusion}

LLLT therapy to treat recalcitrant chalazia demonstrated excellent results, with $39 \%$ of eyes showing resolution after a single 15 min treatment combined with topical and/or oral pharmaceutical therapy; $91 \%$ of eyes showed resolution after one or two treatments, all within two months. 


\section{Acknowledgments}

Physicians Protocol (KGS) provided funding to Science in Vision to assist with data analysis and writing. Sarah Makari of Science in Vision aided in the preparation of this manuscript.

\section{Disclosure}

Dr Karl Stonecipher is a consultant to Eyevance, Eyepoint, J and J, Kala, Nidek, Novartis, Presbia, Refocus, Espansione Marketing S.p.A., Alcon, Allergan, and Bausch Health. He reports personal fees from Allergan, Alcon, Espansione, and Bausch Health during the conduct of the study. He also received grants and personal fees from Allergan, Alcon, Bausch Health, Presbia, Refocus, and Espansione, outside the submitted work. He is an employee of TLC. Dr Rick Potvin reports personal fees from Physicians Protocol during the conduct of the study. The authors report no other conflicts of interest in this work.

\section{References}

1. Jordan GA, Beier K. Chalazion 2019 Mar 13. StatPearls [internet]. Treasure Island (FL): StatPearls Publishing; 2019 January. Available from: http://www.ncbi.nlm.nih.gov/books/NBK499889/PubMed. PMID: 29763064

2. Gilchrist H, Lee G. Management of chalazia in general practice. Aust Fam Physician. 2009;38(5):311-314.

3. Cottrell DG, Bosanquet RC, Fawcett IM. Chalazions: the frequency of spontaneous resolution. $\mathrm{Br}$ Med $J$ (Clin Res Ed). 1983;287 (6405):1595. doi:10.1136/bmj.287.6392.585-a

4. Aycinena AR, Achiron A, Paul M, Burgansky-Eliash Z. Incision and curettage versus steroid injection for the treatment of chalazia: a metaanalysis. Ophthalmic Plast Reconstr Surg. 2016;32(3):220-224. doi:10.1097/IOP.0000000000000483

5. Mester E, Szende B, Gartner P. The effect of laser beams on the growth of hair in mice. Radiobiol Radiother (Berl). 1968;9:621-626.

6. Lee SY, Park KH, Choi JW, et al. A prospective, randomized, placebocontrolled, double-blinded, and split-face clinical study on LED phototherapy for skin rejuvenation: clinical, profilometric, histologic, ultrastructural, and biochemical evaluations and comparison of three different treatment settings. J Photochem Photobiol B. 2007;88(1):51-67. doi:10.1016/j. jphotobiol.2007.04.008

7. Avci P, Gupta A, Sadasivam M, et al. Low-level laser (light) therapy (LLLT) in skin: stimulating, healing, restoring. Semin Cutan Med Surg. 2013;32(1):41-52.

8. de Almeida P 1, Lopes-Martins RA, De Marchi T, et al. Red (660 nm) and infrared $(830 \mathrm{~nm})$ low-level laser therapy in skeletal muscle fatigue in humans: what is better? Lasers Med Sci. 2012;27(2):453458. doi:10.1007/s10103-011-0957-3

9. Kim WS, Calderhead RG. Is light-emitting diode phototherapy (LEDLLLT) really effective? Laser Ther. 2011;20(3):205-215. Review. PubMed PMID: 24155530; PubMed Central PMCID: PMC3799034.
10. Sommer AP. Mitochondrial cytochrome c oxidase is not the primary acceptor for near infrared light-it is mitochondrial bound water: the principles of low-level light therapy. Ann Transl Med. 2019;7 (Suppl1):S13. doi:10.21037/atm.2019.01.43

11. Kipshidze N, Nikolaychik V, Keelan MH, et al. Low-power helium: neon laser irradiation enhances production of vascular endothelial growth factor and promotes growth of endothelial cells in vitro. Lasers Surg Med. 2001;28:355-364. doi:10.1002/1sm.1062

12. Walski T, Dąbrowska K, Drohomirecka A, et al. The effect of Red-toNear-Infrared (R/NIR) irradiation on inflammatory processes. Int $J$ Radiat Biol. 2019;6:1-37.

13. Stonecipher K, Abell TG, Chotiner B, Chotiner E, Potvin R. Combined low level light therapy and intense pulsed light therapy for the treatment of meibomian gland dysfunction. Clin Ophthalmol. 2019;13:993-999. doi:10.2147/OPTH.S213664

14. Toyos R, Briscoe D, Toyos M. The effects of red light technology on dry eye disease due to meibomian gland dysfunction. JOJ Ophthal. 2017;3(5):555624.

15. Wu AY, Gervasio KA, Gergoudis KN, Wei C, Oestreicher JH, Harvey JT. Conservative therapy for chalazia: is it really effective? Acta Ophthalmol. 2018;96(4):e503-e509. doi:10.1111/aos.13801

16. Jackson TL, Beun L. A prospective study of cost, patient satisfaction, and outcome of treatment of chalazion by medical and nursing staff. Br J Ophthalmol. 2000;84(7):782-785. doi:10.1136/bjo.84.7.782

17. Lee JW, Yau GS, Wong MY, Yuen CY. A comparison of intralesional triamcinolone acetonide injection for primary chalazion in children and adults. Sci World J. 2014;2014:413729. doi:10.1155/2014/413 729

18. Ben Simon GJ, Huang L, Nakra T, Schwarcz RM, McCann JD, Goldberg RA. Intralesional triamcinolone acetonide injection for primary and recurrent chalazia: is it really effective? Ophthalmology. 2005;112(5):913-917. doi:10.1016/j.ophtha.2004.11.037

19. Singhania R, Sharma $N$, Vashisht $S$, Dewan $T$. Intralesional Triamcinolone Acetonide (TA) versus Incision and Curettage (I \& C) for medium and large size chalazia. Nepal J Ophthalmol. 2018;10 (19):3-10. doi:10.3126/nepjoph.v10i1.21661

20. Odugbo OP, Mpyet CD, Wade PD, Adenuga OO, Adejoh MO. An audit of minor ophthalmic surgical interventions in a tertiary eye care facility in northern Nigeria. $J$ West Afr Coll Surg. 2014;4(2):26-46.

21. Goawalla A, Lee V. A prospective randomized treatment study comparing three treatment options for chalazia: triamcinolone acetonide injections, incision and curettage and treatment with hot compresses. Clin Exp Ophthalmol. 2007;35(8):706-712. doi:10.1111/j.1442-90 71.2007.01617.x

22. McMonnies CW, Korb DR, Blackie CA. The role of heat in rubbing and massage-related corneal deformation. Cont Lens Anterior Eye. 2012;35(4):148-154. doi:10.1016/j.clae.2012.01.001

23. Ben Simon GJ, Rosen N, Rosner M, Spierer A. Intralesional triamcinolone acetonide injection versus incision and curettage for primary chalazia: a prospective, randomized study. Am J Ophthalmol. 2011;151(4):714-718.e1. doi:10.1016/j.ajo.2010.10.026

24. Knezevic T, Ivekovic R, Astalos JP, Novak Laus K, Mandic Z, Matejcic A. Botulinum toxin A injection for primary and recurrent chalazia. Graefes Arch Clin Exp Ophthalmol. 2009;247(6):789-794. doi:10.1007/s00417-008-0992-3

25. Gaby AR. Nutritional therapies for ocular disorders: part three. Altern Med Rev. 2008;13(3):191-204. 


\section{Publish your work in this journal}

Clinical Ophthalmology is an international, peer-reviewed journal covering all subspecialties within ophthalmology. Key topics include: Optometry; Visual science; Pharmacology and drug therapy in eye diseases; Basic Sciences; Primary and Secondary eye care; Patient Safety and Quality of Care Improvements. This journal is indexed on PubMed

Central and CAS, and is the official journal of The Society of Clinical Ophthalmology (SCO). The manuscript management system is completely online and includes a very quick and fair peer-review system, which is all easy to use. Visit http://www.dovepress.com/ testimonials.php to read real quotes from published authors. 